\title{
GRB PRECURSORS IN THE FALLBACK COLLAPSAR SCENARIO
}

\author{
Xiang-Yu Wang ${ }^{1,2}$, Peter MÉSzÁros ${ }^{1,3}$ \\ Draft version October 4, 2018
}

\begin{abstract}
Precursor emission has been observed in a non-negligible fraction of gamma-ray bursts. The time gap between the precursor and the main burst extends in some case up to hundreds of seconds, such as in GRB041219A, GRB050820A and GRB060124. Both the origin of the precursor and the large value of the time gap are controversial. Here we investigate the maximum possible time gaps arising from the jet propagation inside the progenitor star, in models which assume that the precursor is produced by the jet bow shock or the cocoon breaking out of the progenitor. Due to the pressure drop ahead of the jet head after it reaches the stellar surface, a rarefaction wave propagates back into the jet at the sound speed, which re-accelerates the jet to a relativistic velocity and therefore limits the gap period to within about ten seconds. This scenario therefore cannot explain gaps which are hundreds of seconds long. Instead, we ascribe such long time gaps to the behavior of the central engine, and suggest a fallback collapsar scenario for these bursts. In this scenario, the precursor is produced by a weak jet formed during the initial core collapse, possibly related to MHD processes associated with a short-lived proto-neutron star, while the main burst is produced by a stronger jet fed by fallback accretion onto the black hole resulting from the collapse of the neutron star. We have examined the propagation times of the weak precursor jet through the stellar progenitor. We find that the initial weak jet can break out of the progenitor in a time less than ten seconds (a typical precursor duration) provided that it has a moderately high relativistic Lorentz factor $\Gamma \gtrsim 10$. The longer $(\sim 100 \mathrm{~s})$ time gap following this is determined, in this scenario, by the fall-back timescale, which is at the same time long enough for the exit channel to close after the precursor activity ceases, thus allowing for the collimation by the cocoon pressure of the subsequent main jet, as required.
\end{abstract}

Subject headings: gamma rays: bursts — radiation mechanisms: non-thermal

\section{INTRODUCTION}

Weak, softer precursor emission, occurring from several seconds to hundreds of seconds before the main gamma-ray pulse, is occasionally detected in long gamma-ray bursts (GRB). Often there is almost no emission detectable in the time gap between the precursor and the main event. The earliest detections of precursor events were made with the Ginga satellite (GRB900126, Murakami et al. 1991). Since then, precursors have been found in many bursts, e.g. by BeppoSAX (e.g. GRB011121, Piro et al. 2005), HETE2 (e.g. GRB030329, Vanderspek et al. 2004), INTEGRAL (e.g. GRB041219A, Mereghetti et al. 2004) and Swift (e.g. GRB050820A, Cenko et al. 2006; GRB060124, Romano et al. 2006; GRB061121, Page et al. 2007). Lazzati (2005) analyzed a sample of bright, long BATSE burst light curves in the $200 \mathrm{~s}$ period before the detection of the GRB prompt emission, and found that a sizable fraction ( $\sim 20 \%$ ) of them show evidence for gamma-ray emission above the background between 10 to $\sim 200 \mathrm{~s}$ (the limit of the investigated period) before the main burst. The precursors are typically characterized by non-thermal spectra which tend to be softer than the main burst, and contain from $0.1 \%$ to a few $\%$ of the total event counts. For some bursts with known redshifts, the isotropic energies of the precursors are determined, and can be as large as $E_{\text {iso }} \simeq 5 \times 10^{51} \mathrm{erg}$ for GRB060124 (Romano et al. 2006; Misra et al. 2007). The time gap between the precursor and the main event ranges from several seconds to a few hundreds of seconds (e.g. $200 \mathrm{~s}$ for GRB041219A and GRB050820A, and 570 s for GRB060124 in the observer frame).

Theoretical models for precursors have been proposed, relating it to the breakout of the main GRB jet. As suggested by Ramirez-Ruiz et al. (2002a) and Waxman \& Mészáros (2003), when the jet is making its way out of the stellar mantle, a bow shock runs ahead and a strong thermal precursor is produced as this shock breaks out ${ }^{4}$. In this model, as usual, the main gamma-ray burst is produced when the internal shock occurs at some radius $R_{\gamma}$, the delay time between precursor and the main burst being $\Delta t \simeq R_{\gamma} / 2 \Gamma^{2} c$, where $\Gamma$ is the Lorentz factor of the jet. Since the bursts with a precursor also display significant $\gtrsim \mathrm{MeV}$ emission, similarly to other typical long bursts, their bulk Lorentz factors must be likewise $\Gamma \gtrsim 100$ (e.g. Baring \& Harding 1997; Lithwick \& Sari 2001), hence the typical delay $\Delta t$ is less then a second for the typical internal shock radii $R_{\gamma} \sim 10^{12}-10^{14} \mathrm{~cm}$ (see Mészáros (2006) and Zhang (2007) for a recent review). Since the variability timescale is also $R_{\gamma} / 2 \Gamma^{2} c$, this scenario would imply

\footnotetext{
${ }^{1}$ Department of Astronomy and Astrophysics, Pennsylvania State University, University Park, PA 16802, USA

${ }^{2}$ Department of Astronomy, Nanjing University, Nanjing 210093, China

${ }^{3}$ Department of Physics, Pennsylvania State University, University Park, PA 16802, USA

${ }^{4} \mathrm{~A}$ non-thermal component could also arise during shock breakout, due to the repeated bulk Compton scattering of shock breakout thermal photons by the relativistic ejecta driving the shock (Wang et al. 2006).
} 
a gap timescale comparable to the variability timescale, which is clearly not the case. Morsony et al. (2007) investigated the jet propagation through the star with an elaborate numerical simulation and identified three distinct phases during the evolution, i.e. the precursor phase, the shocked jet phase and the unshocked jet phase. They find that a high-pressure cocoon, formed as the sub-relativistic jet head makes its way out of the star, is released when the head of the jet reaches the stellar surface (which can produce a precursor, Ramirez-Ruiz et al. 2002b). Within the framework of this model, an observer which is located at small or modest viewing angles relative to the jet axis would be expected to see first a relatively bright precursor, then a gap phase during which there is little emission, and finally a bright GRB phase when the unshocked jet reaches the radius of the stellar surface.

In $\S 2$, we consider first a precursor model such as discussed above, which is based on the time delays associated with the same jet giving rise both to the precursor and to the main burst event. We show that the shocked jet phase could not last much longer than $\sim 10 \mathrm{~s}$, due to the fact that once the jet head reaches the stellar surface, a rarefaction wave will form which propagates back into the shocked jet component, decreasing the pressure in the shocked jet significantly, and as a result, the jet attains its ultimate relativistic velocity. Hence, the propagation time taken by the unshocked jet to reach the stellar surface will be limited to $\sim R_{\star} / c$, which is only from a few seconds to ten seconds for Wolf-Rayet progenitors of long GRBs, where $R_{\star}$ is the stellar radius of the progenitor. Thus it appears that this model has difficulties in explaining the $\sim 100 \mathrm{~s}$ long gaps seen in some GRBs. In $\S 3$ we then suggest that gaps hundreds of seconds long could arise in a fallback collapsar scenario, or in a "type II collapsar" scenario (MacFadyen et al. 2001), the gap time being related to the feeding time of the fallback disk, or the instability timescale in the accretion disk. We argue that in such scenarios an initial weak jet, which is still able to break out through the star, produces the precursor, while the main burst is produced by a subsequent more energetic jet, fed by accretion of the fallback gas. The initial precursor jet, whatever its formation mechanism, must be weak enough not to disrupt the star, so that a fallback disk can still form later on. This constrains the luminosity of the precursor jet to be lower than $E_{\mathrm{b}} / t_{j}$, where $E_{\mathrm{b}} \simeq 10^{51} \mathrm{erg}$ is the binding energy of the star envelope and $t_{j}$ is the precursor duration. Motivated by the findings of Woosley \& Zhang (2007) that a weaker jet propagates more slowly inside the jet, in $\S 4$ we study analytically the dependence of the propagation time of the jet on other jet parameters. We confirm here the findings of these simulations, and obtain some constraints on the precursor jet luminosity and Lorentz factors. Some possible formation mechanisms for the precursor jet are considered in $\S 5$. A summary of the results is given in $\S 6$.

\section{JET DYNAMICS INSIDE THE STAR AND AT THE BREAKOUT}

In the collapsar model (Woosley 1993; Paczyński 1998; MacFadyen \& Woosley 1999), GRBs are caused by relativistic jets expelled along the rotation axis of a collapsing stellar core. The relativistic jets are due to a black hole or a neutron star accretion disk, after the iron core of the massive star progenitor has collapsed.

As the jet advances through the star, it drives a bow shock ahead of itself into the star, while the ram pressure of the shocked gas ahead of the jet drives a reverse shock into the head of the jet, slowing the jet head down to a subrelativistic velocity. Thus there are three distinct regions: 1) In front of the contact discontinuity between the jet and the stellar gas, there is a thin layer of shocked stellar gas moving ahead with a sub-relativistic velocity $\sim v_{h}$ into the star; 2) behind the contact discontinuity, there is a shocked jet region, where the relativistic jet with $\Gamma_{j} \gg 1$, is slowed down to a velocity $\sim v_{h}$ by the reverse shock, and 3) and below this is the unshocked jet, whose bulk Lorentz factor behaves as if it were a free jet. In this unshocked jet, at lower radii, the gradual conversion of its internal energy into kinetic energy results in $\Gamma_{j}=\Gamma_{0}\left(r \theta_{j} / r_{0} \theta_{0}\right)$, until a saturation radius at which it reaches its asymptotic value. Here $\theta_{j}$ is the opening angle of the jet at radius $r$ and $\Gamma_{0}$ and $\theta_{0}$ are, respectively, the initial Lorentz factor and opening angle at the injection radius $r_{0}$.

Let us first consider the scenario in which the precursor is related to the jet breakout (e.g. Ramirez-Ruiz et al. 2002a,b; Waxman \& Mészáros 2003; Morsony et al. 2007). In this scenario, the precursor is produced by the bow shock emission or the cocoon emission when the jet head breaks out of the stellar surface. After this, the shocked jet phase emerges, which may have a relatively small opening angle for ultra-relativistic material due to that the mixing of the shocked jet with stellar material lowers its terminal Lorentz factor, so that an observer located at a larger viewing angle would see a dark phase. The relativistic reverse shock may be located well below the stellar surface at the time when the jet head has just reached the surface. One may think that this dark phase could last long enough, if the shocked jet material moves with a low velocity. However, once the jet head reaches the stellar surface, the pressure in front of the jet head decreases suddenly and a rarefaction wave will form and propagate back into the shocked jet material at the speed of sound. The speed of sound in the shocked jet plasma is relativistic, $c_{s}=c / \sqrt{3}$, even if the head velocity $v_{h} \ll c$. When this rarefaction wave arrives at the reverse shock, the pressure of the shocked jet material also drops and it can no longer decelerate the fast unshocked jet. Supposing that the reverse shock is located at a position well below the stellar surface when the jet head reaches the stellar surface, the width of the shocked jet is $\Delta \lesssim R_{\star}$, and the time that the rarefaction wave takes to arrive at the reverse shock is $t_{1}=\Delta / c_{s} \lesssim R_{\star} / c_{s}=6 R_{\star, 11} \mathrm{~s}$. A new system of forward and reverse shocks will form, and the rarefied shocked jet plasma is accelerated to a relativistic velocity with a Lorentz factor

$$
\Gamma_{h 2}=\Gamma_{h 1}\left[4 \Gamma_{h 1}\left(1+c_{s} t / \Delta\right)^{3}\right]^{1 / 4}
$$

where $\Gamma_{h 1}$ is the Lorentz factor of the shocked jet before the rarefaction wave propagating back (see Eq.(15) of Waxman \& Mészáros 2003). Even for a sub-relativistic velocity $\Gamma_{h 1} \simeq 1$, the rarefied shocked jet is accelerated to a relativistic velocity with $\Gamma_{h 2} \gtrsim 2$ after a time $t_{1}$. The unshocked jet then takes a time $t_{2} \simeq R_{\star} / c=3 R_{\star, 11} \mathrm{~s}$ to reach the stellar surface. Supposing that the internal shock occurs at $R_{\gamma}$ where it produces the burst emission, 
the additional delay relative to the precursor emission is $t_{3}=R_{\gamma} / 2 \Gamma^{2} c$. Therefore the total gap period between the precursor and the main burst would be only

$$
T_{\text {gap }}=t_{1}+t_{2}+t_{3} \simeq 10 R_{\star, 11} \mathrm{~s} .
$$

\section{FALLBACK COLLAPSAR SCENARIO}

The above described difficulty in interpreting long $(\sim$ $100 \mathrm{~s}$ ) precursor gaps through a single jet breakout makes it necessary to explore other alternatives. Here we propose a model where the gap is related to the central engine activity, and the precursor is produced by an initial weak jet launched before the main jet. The $\sim 100$ s long gap is remiscent of the natural timescales calculated numerically in the fallback collapsar (type II collapsar) model (MacFadyen et al. 2001), where the fallback disk forms minutes to hours after the initial core collapse ${ }^{5}$. In this scenario, the collapse of the iron core initially forms a proto-neutron star and launches a supernova shock. However, this shock lacks sufficient energy to eject all the matter outside the neutron star, especially for more massive helium cores, because the gravitational binding energy of the helium core increases with mass roughly quadratically, while the explosion energy decreases (Fryer 1999). If the explosion energy is lower than $10^{51} \mathrm{erg}$, as the supernova shock decelerates while it travels outward, some of the expanding material can decelerate below the escape velocity and falls back.

During the initial collapse, it is possible that a weak jet with an energy of, say, a few times $10^{50} \mathrm{erg}$, is produced as a result of MHD processes in the collapsed core, or through propeller effects associated with a proto-neutron star, etc. (e.g. Wheeler et al, 2000). If such a jet is weak enough so as not to immediately disrupt the star, a fallback accretion disk can form in the core on a fallback timescale, which can launch a stronger jet producing the main burst. We argue that such initial weak jets may be responsible for precursors with non-thermal spectra, through internal dissipation mechanisms such as internal shocks or reconnection, after they exit the stellar progenitor. The nonthermal spectra are consistent with those observed from most GRB precursors (e.g. Lazzati 2005; Cenko et al. 2006; Romano et al. 2006; Page et al. 2007).

After a time $\sim 100 \mathrm{~s}$ following the initial collapse, in the case of a weak supernova, enough gas has fallen back for the neutron star to collapse to a black hole. Gas that continues to fall back with sufficient angular momentum will settle into a disk (MacFadyen et al. 2001). The blackhole accretion disk system may then produce stronger jets which, through neutrino annihilation or MHD processes (e.g. Blandford-Znajek mechanism), can power the main burst event.

\section{JET PROPAGATION THROUGH THE STELLAR PROGENITOR}

We investigate now whether a weak precursor jet of luminosity $L_{j} \sim 10^{49} \mathrm{ergs}^{-1}$ lasting for $\sim 10$ seconds (the precursor duration) can break out of the star. Previous analytic studies on the jet dynamic inside the star and the jet breakout time have been done by, e.g. Mészáros \& Rees 2001; Ramirez-Ruiz et al. (2002b); Matzner (2003), Lazzati \& Begelman (2005) and Toma et al. (2006). As recently found by Woosley \& Zhang (2007) in the simulation of jet propagation through the star, a lower-energy jet takes a longer time to break out of the star. A jet with a lower Lorentz factor may also take a longer time, while at present there is no constraint yet on the Lorentz factor of the precursors jets since their spectra do not display significant $\gtrsim \mathrm{MeV}$ emission. Since the precursor lifetime is usually only about ten seconds and the breakout time must be shorter than this (otherwise the jet will be chocked), it is not obvious whether it can really break out of the star or not. We will show below that, for a low-energy jet to be able to break out of the star in a time shorter than the precursor duration, it must have a moderately high asymptotic Lorentz factor, $\eta=L / \dot{M} c^{2} \gtrsim 10$. The asymptotic Lorentz factor of this weak precursor jet can, however, be lower than that of the typical main GRB ejecta, which are characterized by $\eta>100$, and this could account for the softer spectrum of the precursor compared to the main burst. Phenomenological relations between the energy (or luminosity) and the spectrum of the burst have been found by e.g. Amati et al. (2002) and Yonetoku et al. (2004), in which a lower energy (or luminosity) burst tends to have a lower peak energy, hence a softer spectrum.

\subsection{Jet crossing time}

The jet head velocity is given by the longitudinal balance between the jet thrust and the ram pressure of material ahead of it (Mészáros \& Rees 2001; Matzner 2003)

$$
v_{h}=\left(\frac{L_{j}}{2 \pi \theta_{j}^{2} r^{2} c \rho}\right)^{1 / 2}=7 \times 10^{9} r_{11}^{1 / 2} L_{j, 49}^{1 / 2} \theta_{j,-2}^{-1} \mathrm{cms}^{-1}
$$

where $\theta_{j}$ is the opening angle of the jet while propagating inside the star, whose value will be derived below, and $\rho$ is the stellar density at radius $r$. The presupernova density profile is assumed to be roughly described by $\rho \propto r^{-3}$ out to the edge of the He core at $r=10^{11} \mathrm{~cm}$ (MacFadyen et al. 2001), at which point we take the density to be $\rho=1 \mathrm{gcm}^{-3}$. The total time taken by the jet head to move from the interior of the star to the surface is

$$
t=\int \frac{d r}{v_{h}}=\frac{\alpha r}{v_{h}}=14 \alpha r_{11}^{1 / 2} L_{j, 49}^{-1 / 2} \theta_{j,-2} \mathrm{~s}
$$

where $\alpha>1$ is an integrating factor over $r$ (discussed after Eq.(10)). While the jet is propagating inside the star with a sub-relativistic velocity, a significant fraction of jet "waste" energy is pumped into the cocoon surrounding the advancing jet. The unshocked jet moving with $\Gamma_{j} \gg 1$ is now constrained in the transverse direction by the pressure of the cocoon, so that it gets collimated and becomes more penetrating. The opening angle $\theta$ of the unshocked jet is determined by the balance between the pressure of the unshocked jet in the transverse direction and that of the cocoon.

The energy stored in the cocoon is roughly $E_{c} \simeq L_{j}(t-$ $r / c)$ when the sub-relativistic jet reaches radius $r$ after a time $t$. The volume of the cocoon is $V_{c}=\frac{\pi}{3} r_{\perp}^{2} r$, where $r_{\perp}=v_{\perp} t$ is the transverse size of the cocoon (Matzner $2003)$. Since the cocoon pressure is much larger than that of the stellar material, the transverse expansion velocity

\footnotetext{
${ }^{5}$ Cheng \& Dai (2001) suggested a similar, "two-step" jet model, which attempts to solve the baryon contamination problem in the hypernova model of GRBs.
} 
$v_{\perp}$ of the cocoon is given by the balance between the cocoon pressure $p_{c}$ and the ram pressure of the stellar material, i.e. $v_{\perp}=\left(p_{c} / \rho\right)^{1 / 2}$. From $p_{c}=E_{c} / 3 V_{c}$, we get

$$
p_{c}=\left(\frac{L_{j} \rho}{\pi r t}\right)^{1 / 2}=\left(\frac{L_{j} \rho v_{h}}{\alpha \pi r^{2}}\right)^{1 / 2} .
$$

The pressure in the unshocked relativistic jet in the transverse direction, from the relativistic Bernoulli equation, is $p_{j, \perp}=\frac{1}{3} L_{j} /\left(2 \pi r^{2} \theta_{j}^{2} c \Gamma_{j}^{2}\right)$. As the internal energy gets converted into the kinetic energy of the jet, $\Gamma_{j}$ increases. Depending on whether the jet Lorentz factor has saturated or not before the jet breaks out of the star, there are two cases for the evolution of $\Gamma_{j}$ : one is the high $\eta$ case (case I), in which $\Gamma_{j} \propto\left(r \theta_{j}\right)=\Gamma_{0}\left(r / r_{0}\right)\left(\theta_{j} / \theta_{0}\right)<\eta$ all along the path (see the left panel of Fig. 1), and the other is the low $\eta$ case (case II) in which $\Gamma_{j}$ has reached its saturation value $\eta$ at some radius inside the star (see the right panel of Fig. 1). The latter case is more easily satisfied when $\eta$ is lower or when the initial injection radius $r_{0}$ is smaller. The jet pressure is different in the two cases, being given by, respectively,

$$
p_{j, \perp}= \begin{cases}L_{j} r_{0}^{2} \theta_{0}^{2} /\left(6 \pi c r^{4} \theta_{j}^{4} \Gamma_{0}^{2}\right) & \Gamma_{j}<\eta(\text { Case I }) ; \\ L_{j} /\left(6 \pi c r^{2} \theta_{j}^{2} \eta^{2}\right) & \Gamma_{j}=\eta(\text { Case II })\end{cases}
$$

From $p_{j, \perp}=p_{c}$, we get the opening angle for the two cases respectively,

$$
\theta_{j}=\left\{\begin{array}{l}
\left(\alpha^{2} L_{j} / 648 \pi \rho c^{3}\right)^{1 / 14}\left(r_{0} \theta_{0} / \Gamma_{0}\right)^{4 / 7} r^{-5 / 7} \\
\left(\alpha^{2} L_{j} / 648 \pi r^{2} \rho c^{3}\right)^{1 / 6} \eta^{-4 / 3}
\end{array}\right.
$$

For case I, with the parameters $\theta_{0}=1, \Gamma_{0}=1, r_{0}=10^{7} \mathrm{~cm}$ and the stellar density profile $\rho=1\left(r / 10^{11} \mathrm{~cm}\right)^{-3} \mathrm{gcm}^{-3}$, the opening angle is

$$
\theta_{j}=1.5 \times 10^{-3} \alpha^{1 / 7} L_{j, 49}^{1 / 14} r_{11}^{-1 / 2} r_{0,7}^{4 / 7},
$$

while for case II,

$$
\theta_{j}=3 \times 10^{-3} \alpha^{1 / 3} L_{j, 49}^{1 / 6} r_{11}^{1 / 6} \eta_{1}^{-4 / 3} .
$$

Note that these values of the opening angles are the ones at the jet breakout time, which are not the final values that we expect in the internal shock phase or in the afterglow. After exiting the star, the hot jet is expected to expand freely to an opening angle comparable to $1 / \Gamma_{\mathrm{br}} \sim 0.1$, where $\Gamma_{b r}$ is the Lorentz factor of the jet at the breakout time.

With the above values of the opening angles, we can obtain the jet breakout time from Eq.(4) for the two cases,

$t_{\mathrm{br}}=\left\{\begin{array}{l}\alpha^{8 / 7} r^{9 / 7}\left(\frac{4 \pi^{3} \rho^{3} c^{2}}{L_{j}^{3}}\right)^{1 / 7}\left(\frac{r_{0} \theta_{0}}{\Gamma_{0}}\right)^{4 / 7}=2 \alpha^{8 / 7} L_{j, 49}^{-3 / 7} r_{0,7}^{4 / 7} \mathrm{~s} \\ \alpha^{4 / 3} r^{5 / 3}\left(\frac{L_{j}}{\pi \rho}\right)^{-1 / 3} \eta^{-4 / 3}=4 \alpha^{4 / 3} r_{11}^{2 / 3} L_{j, 49}^{-1 / 3} \eta_{1}^{-4 / 3} \mathrm{~s}\end{array}\right.$

Here $\alpha$ is the integrating factor over $r$ in Eq.(4). In case I, since $\rho \propto r^{-3}$ starts roughly above $r=10^{9} \mathrm{~cm}$, below which the free fall density profile $\rho \propto r^{-3 / 2}$ may apply (Mészáros \& Rees 2001), $\alpha={ }_{1} \ln \left(r / 10^{9} \mathrm{~cm}\right) \simeq 4$ for $r=10^{11} \mathrm{~cm}$. In case II, the integration over $r$ in Eq.(4) gives $\alpha_{2}=3 / 2$. We can see that a low-luminosity, weak jet takes a longer time to break out of the star, consistent with recent findings by Woosley \& Zhang (2007) from the numerical hydrodynamic simulation of the jet propagation. The dependence of the breakout time $t_{\mathrm{br}}$ on the luminosity is illustrated in the inset plot of the left panel of Fig.1. Requiring that the breakout time $t_{\mathrm{br}}$ is less than the duration of the precursor $T_{p} \simeq 10 \mathrm{~s}$, we get, for case $\mathrm{I}$,

$$
L_{j} \gtrsim 10^{49}\left(\alpha_{1} / 4\right)^{8 / 3} r_{0,7}^{4 / 3} T_{p, 1}^{-7 / 3} \mathrm{ergs}^{-1} .
$$

The asymptotic Lorentz factor $\eta$ in this case is required to be larger than $\Gamma_{\mathrm{br}}=\Gamma_{0}\left(r \theta_{j} / r_{0} \theta_{0}\right) \simeq 15$, from the high $\eta$ assumption of case I. For case II, the breakout time is sensitive to $\eta$, more than to the jet luminosity, and we get

$$
\eta \gtrsim 10\left(\alpha_{2} / 1.5\right) r_{11}^{1 / 2} L_{j, 49}^{-1 / 4} T_{p, 1}^{-3 / 4}
$$

by requiring the breakout time to be shorter than $T_{p} \simeq 10 \mathrm{~s}$ (see the inset plot of the right panel of Fig.1). Therefore we see that in both cases, it is only when $\eta \gtrsim 10$ that the jet breakout time can be $\lesssim 10$ seconds, for a luminosity of the order of $L_{j} \sim 10^{49} \mathrm{ergs}^{-1}$. This reflects the fact that a lower Lorentz factor jet is less penetrating and needs a longer time to break out of the stellar progenitor. This shows that even the precursor jet should have a relatively high Lorentz factor, although it need not be as large as $\gtrsim 100$ as in the case of the main burst.

\subsection{Can the precursor channel close up again?}

After the weak jet breaks out, the cocoon material escapes. There is now a wide funnel opened by the weak jet and its accompanying cocoon along its path. As evidenced by GRB050820A (Cenko et al. 2006), a burst with precursor, the main burst jet is collimated to $\theta \sim 0.1$, like other typical long bursts without precursors. Since a closed channel is a necessary condition for the main jet to be collimated, one may ask whether the initial channel, made by the precursor jet, will have had time to close again by the time that the main jet starts to make its way out, $\sim 100$ seconds later.

As the weak jet propagates outward, the cocoon pushes the stellar material sideways. The opening angle of the cocoon, which is also the funnel opening angle after the cocoon flows out, is approximately given by

$$
\theta_{f}=\theta_{c}=\frac{r_{\perp}}{r}=\left(\frac{L_{j} t^{3}}{\pi \rho r^{5}}\right)^{1 / 4}=\alpha \Gamma_{\mathrm{br}}^{-1},
$$

where the last equality is obtained with the help of the second line of Eq.(10). For $\Gamma_{\mathrm{br}} \simeq 10$, the cocoon opening angle is about tens of degrees.

The cocoon can drive a transverse shock into the stellar material along its passage, heating it up, so the pressure of the shocked stellar material $\left(p_{e} \sim \rho v_{\perp}^{2}\right)$ is comparable to that of the cocoon, i.e. $p_{e} \simeq p_{c}$. After the cocoon flows out, this external stellar material will re-expand back into the funnel at a speed of sound given by

$$
c_{s}=\sqrt{p_{e} / \rho} \simeq \sqrt{p_{c} / \rho}=v_{\perp} .
$$

This means that in a time comparable to the precursor jet breakout time, the channel closes up again. From Fig.5 of Lazzati (2005), it is known that the precursor duration is always shorter than the gap period of time. Therefore, we conclude that the channel opened by the precursor jet is 
indeed closed again before the main jet starts to propagate out.

The free-fall time of the material along the rotation axis is

$$
t_{\mathrm{ff}}=3000\left(\frac{\rho}{1 \mathrm{gcm}^{-3}}\right)^{-1 / 2} \mathrm{~s} .
$$

For the $\mathrm{C} / \mathrm{O}$ core material of the collapsed progenitor, whose density is $\rho \sim 500 \mathrm{gcm}^{-3}$, its free-fall time is comparable to the $\sim 100$ s gap period and therefore this material will be essentially evacuated. However, for He core material with $\rho \sim 1 \mathrm{gcm}^{-3}$, the free-fall time is much longer than the gap period, and the funnel in this part (aside from reclosing) remains almost the same as before the collapse, by the time that the main jet starts to propagate out.

\subsection{Transient thermal emission from the open funnel}

The channel made by the initial precursor jet can, while it remains open, produce an interesting emission signature. As the cocoon expands transversally into the stellar material, it will drive a shock. Since this shock takes place in the highly optically thick interior of the progenitor star, it will become a radiation-dominated shock, similarly to the case of the supernova shock propagating inside the star (Weaver 1976). The velocity of the shock driven by the cocoon into the He core material can be derived from $v_{s}=$ $\left(p_{c} / \rho\right)^{1 / 2}=v_{\perp}$, where $p_{c}$ is given by Eq.(5). This gives a shock velocity $v_{s} \simeq 10^{9} \mathrm{cms}^{-1} L_{j, 49}^{3 / 8} \theta_{j,-1}^{-1 / 4} r_{11}^{-3 / 4} \rho^{-3 / 8} \alpha^{-1 / 4}$. For a radiation-dominated shock, this corresponds to a radiation temperature of

$$
T_{r} \sim\left(\frac{L_{j} \rho v_{h}}{a^{2} \alpha \pi r^{2}}\right)^{1 / 8} \sim 8 \alpha^{-1 / 8} L_{j, 49}^{1 / 8}\left(\frac{v_{h}}{c}\right)^{1 / 8} r_{11}^{-1 / 4} \mathrm{KeV}
$$

where $a$ is the Boltzmann energy density constant of radiation.

To check the self-consistency of having assumed a radiation-dominated shock, we need to compare the the radiation diffusion length $\Delta_{\gamma}=c /\left(3 n_{e} v_{s} \sigma_{\mathrm{T}}\right)$ (where $n_{e}$ is the electron number density of the plasma) with the stopping distance of the ions due to Coulomb collisios $l$. According to Weaver \& Chapline (1974), the Coulomb friction is the dominant dissipation mechanism controlling the shock width (i.e. the shock is collisional), provided that the stopping distance of the ions $l$ is larger than the radiation diffusion length $\Delta_{\gamma}$. Otherwise, the shock is radiation-dominated. For an electron temperature $T_{e}=T_{r}$, the ion-electron collision rate is $\nu_{i e}=2 \times$ $10^{10}\left(\rho / 1 \mathrm{gcm}^{-3}\right)\left(T_{e} / 10 \mathrm{KeV}\right)^{-3 / 2} \mathrm{~s}^{-1}$, while the ion-ion collision rate, for an ion temperature of $T_{i}=(3 / 16) m_{p} v_{s}^{2}=$ $200 \mathrm{KeV}$, is $\nu_{i i}=1 \times 10^{10}\left(\rho / 1 \mathrm{gcm}^{-3}\right)\left(T_{i} / 200 \mathrm{KeV}\right)^{-3 / 2} \mathrm{~s}^{-1}$ (Spitzer 1962; Waxman \& Loeb 2001). Thus, the stopping of the ions is dominated by interaction with the electrons, with a mean free path given by $l_{i e}=\nu_{i e}^{-1} v_{s}=$ $0.05 \mathrm{~cm}\left(\rho / 1 \mathrm{gcm}^{-3}\right)^{-1}\left(T_{e} / 10 \mathrm{KeV}\right)^{3 / 2}\left(v_{s} / 10^{9} \mathrm{cms}^{-1}\right)$. The radiation diffusion length is $\Delta_{\gamma}=20 \mathrm{~cm}\left(\rho / 1 \mathrm{gcm}^{-3}\right)^{-1}\left(v_{s} / 10^{9}\right.$ Since $\Delta_{\gamma} \gg l_{i e}$, the scale (width) of the shock is determined by $\Delta_{\gamma}$. Except for a thin layer at the leading edge of the shock front, the Coulomb friction is unimportant, and the shock is effectively radiation-dominated. For a radiation temperature of $T_{r} \simeq 10 \mathrm{KeV}$, Compton scattering gives a cooling rate of $t_{\mathrm{Comp}}^{-1}=\left(8 \sigma_{T} / 3 m_{e} c\right) a T_{r}^{4}=$ $8 \times 10^{10}\left(T_{r} / 10 \mathrm{KeV}\right)^{4} \mathrm{~s}^{-1}$, while the Bremsstrahlung cooling rate is $t_{\text {Brem }}^{-1}=3 \times 10^{8}\left(\rho / 1 \mathrm{gcm}^{-3}\right)\left(T_{e} / 10 \mathrm{KeV}\right)^{-1 / 2} \mathrm{~s}^{-1}$. Thus, Compton scattering also provides the dominant mechanism for cooling the electrons, and this can reduce the ion temperature significantly from those that would occur in a Coulomb viscosity-dominated shock. These photons diffuse ahead, creating sufficient pressure to decelerate the electrons (as viewed in the frame of the shock front) and the ions are then decelerated by these electrons through Coulomb friction. We note that the above rough quantitative argument is only an approximate treatment of this issue. A more detailed numerical treatment taking into account the energy and momentum balance in the shock transition as well as relativistic effects $^{6}$, as performed by Weaver (1976), is beyond the scope of the present work. However, there is a useful numerical criterion for checking the self-consistency of a radiation-dominated shock. According to Weaver (1976), the radiative heat transport suffices for producing the necessary dissipation in a strong shock wave if the ratio of the radiation pressure to matter pressure $P_{r} / P_{m}$ exceeds 4.45 . This condition is satisfied in our case, the ratio of the two pressures being $P_{r} / P_{m}=a T_{r}^{4} / n_{e} k T_{e}=\rho v_{s}^{2} / n_{e} k T_{r} \simeq 100$ for $v_{s}=10^{9} \mathrm{cms}^{-1}$ and $T_{e}=T_{r}=10 \mathrm{KeV}$, justifying the assumption of a radiation-dominated shock.

The shock-heated walls of the jet channel, before the latter closes up again, can radiate a transient thermal pulse with characteristic photon energy $k T \sim 10 \mathrm{KeV}$ and a luminosity

$$
\begin{aligned}
& L_{X} \simeq \sigma T^{4} \pi\left(R_{\star} \theta_{f}\right)^{2} \\
& =2 \times 10^{48} \alpha^{3 / 2} L_{j, 49}^{1 / 2}\left(\frac{v_{h}}{c}\right)^{1 / 2} R_{\star, 11} \eta_{1}^{-2} \mathrm{ergs}^{-1}
\end{aligned}
$$

As the cocoon escapes from the channel, the cocoon pressure decreases significantly, as does the radiation energy density of the photon field. Finally, as the pressure wanes, the channel closes up again under the pressure of the external stellar material. The emission from the channel walls vanishes once the channel has reclosed, so this transient thermal pulse is expected to last for a time comparable to the jet crossing time given by Eq.(10). This could appear as a thermal precursor of $\sim 10$ seconds duration, besides the non-thermal emission component from the cocoon itself.

\section{POSSIBLE FORMATION MECHANISMS OF THE PRECURSOR JETS}

How does the weak precursor jet form during the initial core collapse? There is a long-standing speculation that when rotation and magnetic fields are taken into account, the core collapse of massive stars can lead to some form of MHD outflows, although it is unknown whether they can power supernovae or gamma-ray bursts (e.g. LeBlance \& Wilson 1970; Wheeler et al. 2000, 2002; Woosley \& cmBtó $\phi \bar{n} t .2006$; Metzger et al. 2007). Following Wheeler et al. (2000), here we consider that a relatively weak jet of energy a few times $10^{50} \mathrm{erg}$ is produced by a rotating protoneutron star during the initial core collapse phase. This is motivated by the consideration that the rotation energy of

${ }^{6}$ For a radiation temperature $T_{r} \simeq 10 \mathrm{KeV}$, the pair production effect is unimportant (Weaver 1974) 
a proto-neutron star with a radius of $50 \mathrm{~km}$ is suitable for GRB precursor jets. Immediately after the core collapse, a proto-neutron star (PNS) forms with a radius of $\sim 50 \mathrm{~km}$ and mass $\sim 1.4 M_{\odot}$. For a specific angular momentum of the progenitor core of $j \simeq 10^{16} \mathrm{~cm}^{-2} \mathrm{~s}^{-1}$, the angular rotation velocity of the PNS is $\Omega \simeq 400 \mathrm{~s}^{-1}$, assuming angular momentum conservation. The rotation energy of the proto-neutron star is

$E_{\mathrm{rot}, \mathrm{PNS}} \simeq \frac{1}{2} I_{\mathrm{PNS}} \Omega^{2} \simeq 2 \times 10^{51}\left(\frac{\Omega}{400 \mathrm{~s}^{-1}}\right)^{2}\left(\frac{R_{\mathrm{PNS}}}{50 \mathrm{~km}}\right)^{2} \mathrm{erg}$

Over a cooling time $\sim 5-10$ s, the hot proto-neutron star deleptonizes by neutrino emission and contracts to form the final neutron star. If a fraction, e.g. $\sim 10 \%$ of the rotation energy is tapped by some MHD process and converted into a relativistic outflow, this outflow will have the right energy and timescale for a GRB precursor. One possible scenario for such an MHD process is that the differentially rotating neutron star can wind up the poloidal seed field inside the proto-neutron star into strong toroidal fields, which then emerge from the star through buoyancy (Kluźnizk \& Ruderman 1998; Dai \& Lu 1998; Dai et al. 2006). Once the field emerges through the stellar surface, it will trigger an explosive reconnection and release the magnetic energy, resulting in a high-entropy fireball. Assuming a critical field value required for buoyancy of $B_{f} \simeq 10^{15} \mathrm{G}$ corresponding to the density of the protoneutron star (Wheeler et al. 2002), the number of revolutions required is $n_{f}=B_{f} / 2 \pi B_{0}=160 B_{f, 15} B_{0,12}^{-1}$ where $B_{0}$ is the magnetic field of the proto-neutron star. The amplification timescale before the field is expelled by buoyancy is thus $\tau_{f} \simeq n_{f} P_{\mathrm{PNS}} \simeq 2.5 B_{f, 15} B_{0,12}^{-1} \mathrm{~s}$, where $P_{\mathrm{PNS}}$ is the spin period of the proto-neutron star.

Note that the infalling matter from the exterior of the proto-neutron star is insufficient to suppress this buoyant magnetic field pressure in a proto-neutron star. The ram pressure at $R_{\mathrm{PNS}}=50 \mathrm{~km}$ for a mass infall rate $\dot{M}$ is

$p_{\mathrm{ram}}=\frac{\dot{M} v_{f f}}{4 \pi R_{\mathrm{PNS}}^{2}} \simeq 5 \times 10^{26} \dot{M}_{-2} M_{0}^{1 / 2}\left(\frac{R_{\mathrm{PNS}}}{50 \mathrm{~km}}\right)^{-5 / 2} \mathrm{ergs}^{-1}$

(Fryer 1999), which is much smaller than the magnetic field pressure $p_{B}=B_{f}^{2} / 8 \pi \simeq 4 \times 10^{28} B_{f, 15}^{2} \mathrm{ergs}^{-1}$, where $v_{f f}=\left(2 G M / R_{\mathrm{PNS}}\right)^{1 / 2}$ is the free-fall velocity, $\dot{M}$ is the mass infalling rate in units of $M_{\odot} \mathrm{s}^{-1}$ and $M_{0}$ is the protoneutron star mass. The infall rate of matter at early times (the first $10 \mathrm{~s}$ ) in the fallback collapsar is uncertain, but as a rough estimate, the accretion rate $\left(\sim 0.01 M_{\odot} s^{-1}\right)$ during the fallback phase (the first $100 \mathrm{~s}$ ) can be used (see Fig.5 of MacFadyen et al. 2001). However, after about 10 s neutrino cooling will tend to shrink the proto-neutron star size, and since the ram pressure is proportional to $R^{-5 / 2}$, even if a magnetar with $B \lesssim 10^{15} \mathrm{G}$ could form after contraction, its activity would be suppressed, due to its smaller size $R$, by the ram pressure of the infalling matter after the infall time $\sim 100 \mathrm{~s}$.

Since the activity of a central neutron star with a magnetic field $B \lesssim 10^{15} \mathrm{G}$ can be suppressed by the infalling matter, no significant emission would be seen after the proto-neutron star phase, until the accretion disk forming at a later time results in the formation of a newborn black hole. In the fallback collapsar scenario, the accretion rate depends on the initial supernova shock energy. For a weak SN explosion with an energy $\lesssim 0.5 \times 10^{51} \mathrm{erg}$, the accretion rate is about $\sim 0.01 M_{\odot} s^{-1}$ (see Fig. 5 of MacFadyen et al. (2001)). With the above accretion rate, the neutron star will collapse to a black hole on a timescale of the order of $30-100$ seconds. After the black hole forms, gas that continues to fall in with sufficient angular momentum will settle into a disk, which can power a main jet through MHD processes or neutrino annihilation in the disk-black hole system. This $\sim 100 \mathrm{~s}$ accumulation timescale of the accretion material may be responsible for the long time delay of inactivity seen in some bursts with precursors.

Another possible mechanism for causing a long-time gap might be the development of instabilities in the hyperaccretion disk (e.g. Perna et al. 2006; Janiuk et al. 2007). Janiouk et al. (2007) found that the opaque inner regions of the disk become unstable to convective motions. This may give rise to the short-term time variability seen in the prompt phase, but can not explain the long-time gap. The long time delay may require, e.g., that the disk is gravitationally unstable, fragmenting into rings. The gravitational instability sets in when the Toomre parameter (Toomre 1964) $Q=c_{s} \kappa / \pi G \Sigma<1$, where $c_{s}$ is the sound speed, $\kappa$ is the local epicyclic frequency and $\Sigma$ is the mass surface density. The disk in the case of a merger of compact objects is found to be gravitationally stable, since $Q>1$, according to Lee \& Ramirez-Ruiz (2002) and Lee et al. (2005). In order to explain the X-ray flares seen in short GRBs, Perna et al. (2006) speculated that the outer part of the disk may become gravitationally unstable, when the accretion rate is as high as $10 \mathrm{M}_{\odot} \mathrm{s}^{-1}$. However, for fallback collapsars, the outer radius at which this criteria is satisfied may lie well outside the the accretion shock radius seen in the fallback calculations by MacFadyen et al.(2001), which casts into doubt the viability of this mechanism for the long-time gap.

The long gap may also be produced by a modulated relativistic wind without postulating any quiet phase in the central engine (Ramirez-Ruiz et al. 2001). It was also pointed out that an approach to test this would be through observations of the prompt afterglow emission from the reverse shock.

\section{DISCUSSION}

We have explored the constraints posed by GRB precursors with long time gaps on the jet dynamics while propagating through the progenitor stars and jet parameters. We showed that a $\sim 100 \mathrm{~s}$ long gap is hard to interpret in terms of a single jet breaking out through the stellar envelope, and we suggested a possible two stage scenario, involving a precursor jet powered by an initial proto-neutron star, and a main burst powered by a fallback collapsar leading to a black hole. In this two stage central engine scenario, the precursor jet in the first stage must be weak, so as not to disrupt the star, otherwise there would be no fallback accretion disk to power the second stage main jet. Since the observed precursor durations are usually short, of the order of ten seconds, the jet propagation time through the star poses severe constraints on the jet physical parameters, such as the jet luminosity and Lorentz factor. One of our aims has been 
to give an analytic calculation of the jet propagation time, in order to explore the properties of precursor gap time models. Comparison of our analytical results with numerical hydrodynamical simulations such as those of Woosley \& Zhang (2007) show that the analytical dependence of the propagation time on the jet luminosity is in qualitative agreement with these simulation results.

We have studied the effects of a precursor jet breaking out from the progenitor and its related possible observational manifestations, within the context of our two-stage scenario. The precursor jet opens a channel through the star as it makes its way out. The channel will re-close after the precursor jet and its cocoon have escaped, on a timescale of $\sim 10 \mathrm{~s}$. This allows a main jet to form as a result of a collapsar black hole accretion, if the precursor is weak enough so as not to disrupt the star, the main jet being collimated by the stellar pressure and propagating outward in the usual manner. These analytical results are approximate, and numerical hydrodynamical simulation studies of such a two-stage jet model would be required to verify its behavior in more detail.

We have considered some of the possible formation mechanisms for the precursor jet in the framework of the two-stage fallback collapsar scenario, involving MHD activity of an initial proto-neutron star. We stress that a proto-neutron star MHD outflow is just one possibility, especially considering the many uncertainties involved both in the proto-neutron star and in the fallback collapsar scenarios. The possible origin of the $\sim 100 \mathrm{~s}$ inactivity of the central engine, in this two-stage fallback collapsar framework, is also tentative. However, for any possible two-stage scenario, the above discussion on the jet-star interaction dynamics remains applicable, and the constraints we have discussed remain applicable.

In summary, we have argued that the long time $(\sim 100$ s) gap between the precursor and the main burst may be attributed to the central engine behavior. One natural scenario resulting in a $\sim 100 \mathrm{~s}$ delay is the fallback collapsar model (MacFadyen et al. 2001). We suggest that the initial precursor jet may arise in an initial stage of the collapsed core, possibly related to MHD processes or neutrino emission occurring in a proto-neutron star. We have calculated the crossing time of the weak precursor jet and have shown that this initial weak jet can break out through the progenitor envelope in a time less than ten seconds, which is a typical precursor duration, provided that it has a relatively high Lorentz factor $\Gamma \gtrsim 10$. The channel opened by the precursor jet along the rotation axis of the collapsar may produce a transient weak thermal precursor, before it recloses due to the re-expanding stellar material which was heated by the cocoon of the precursor jet. This provides the conditions required for the collimation of the main jet, which starts out after a typical fall-back timescale of $\sim 100$ seconds, producing a black hole leading to the main GRB.

We thank the referee for incisive and helpful reports which have led to significant improvements of the manuscript. This work is supported in part by NASA NAG5-13286, NSF AST 0307376, and the National Natural Science Foundation of China under grants 10403002 and 10221001, and the Foundation for the Authors of National Excellent Doctoral Dissertations of China (for X.Y.W.).

\section{REFERENCES}

Amati, L., 2002, A\&A, 390, 81

Baring M. G., \& Harding, A. K. 1997, ApJ, 491, 663

Cenko, S. B. et al. 2006, ApJ, in press, astro-ph/0608183

Cheng, K. S. \& Dai, Z. G., 2001, Astroparticle Physics, 16, 67

Dai, Z. G. \& Lu, T. 1998, Phys. Rev. Lett. 81, 4301

Dai, Z. G., Wang, X. Y., Wu, X. F. \& Zhang, B. 2006, Science, 311, 1127

Fryer, C. L. 1999, ApJ, 522, 413

Janiuk, A. Yuan, Y.-F., Perna, R. \&di Matteo,T. 2007, ApJ, in press, arXiv:0704.1325

Kluźnizk, W. \& Ruderman, M. 1998, ApJ, 505, L113

Lazzati, D. 2005, MNRAS, 357, 722

Lazzati, D. \& Begelman, M. C. 2005, ApJ, 629, 903

LeBlanc, J. H. \& Wilson, J. R. 1970, ApJ, 161, 541

Lee, W. H., \& Ramirez-Ruiz, E. 2002, ApJ, 577, 893

Lee, W. H., Ramirez-Ruiz, E. \& Page, D., 2005, ApJ, 632, 421

Lithwick, Y. \& Sari, R., 2001, ApJ, 555, 540

MacFadyen, A. I.\& Woosley, S. E., 1999, ApJ, 524, 262

MacFadyen, A. I., Woosley, S. E. \& Heger, A. 2001, ApJ, 550, 410

Metzger, B. D., Thompson, T. A. \& Quataert, E. 2007, ApJ, in press, astro-ph/0608682

Matzner, C. D. 2003, MNRAS, 345, 575

Murakami, T., Inoue, H., Nishimura, J. et al. 1991, Nature, 350, 592

Mereghetti, S., Shaw, S., Beck, M. \& Borkowski, J. 2004, GRB Circ. 2866 .

Spitzer, L., 1962, Physics of Fully Ionized Gass (New York: Interscience)

Mészáros , P. \& Rees, M. J., 2001, ApJ, 556, L37

Mészáros , P. 2006, Rep. Prog. Phys., 69, 2259

Morsony, B. J., Lazzati, D. \& Begelman M. C., ApJ, submitted, astro-ph/0609254
Misra, K. et al. 2007, A\&A accepted, astro-ph/0701413

Paczyński B. 1998, ApJ, 494, L45

Page, K. et al., 2007, ApJ, submitted

Perna, R. Armitage, P. J., \& Zhang, B. 2006, 636, L29

Piro, L. et al. 2005, ApJ, 623, 314

Ramirez-Ruiz, E., MacFadyen, A. I., \& Lazzati, D. 2002a, MNRAS, 331, 197

Ramirez-Ruiz, E., Celotti, A. \& Rees, M. J., 2002b, MNRAS, 337, 1349

Ramirez-Ruiz, E., Merloni, A.\& Rees, M. J., 2001, MNRAS, 324, 1147

Romano, P. et al. 2006, A\&A 456, 917

Toma, K., Ioka, K., Sakamoto, T. \& Nakamura T., 2006, ApJ, astro$\mathrm{ph} / 0610867$

Vanderspek, R. et al. 2004, ApJ, 617, 1251

Wang, X. Y., Li, Z., Waxman, E. \& Mészáros , P. 2007, ApJ, 664, 1026

Waxman, E. \& Loeb, A., 2001, Phys. Rev. Lett., 8, 071101

Waxman, E. \& Mészáros, P. 2003, ApJ, 584, 390

Weaver, T. A. \& Chapline, G. F., 1974, ApJ, 192, L57.

Weaver, T. A., 1976, ApJS, 32, 233

Wheeler, J. C., Yi, I., Höflich, P. \& Wang, L. 2000, ApJ, 537, 810

Wheeler, J. C., Meier, D. L., Wilson, J. R., 2002, ApJ, 568, 807

Woosley, S. 1993, ApJ, 405, 273

Woosley, S. \& Bloom, J. S. 2006, ARA\&A, 44, 507

Woosley, S. \& Zhang, W. 2007, proceedings of Royal Society meeting on GRB, sastro-ph/0701320

Yonetoku, D. 2004, ApJ, 609, 935

Zhang, B. 2007, ChJAA, 7, 1 

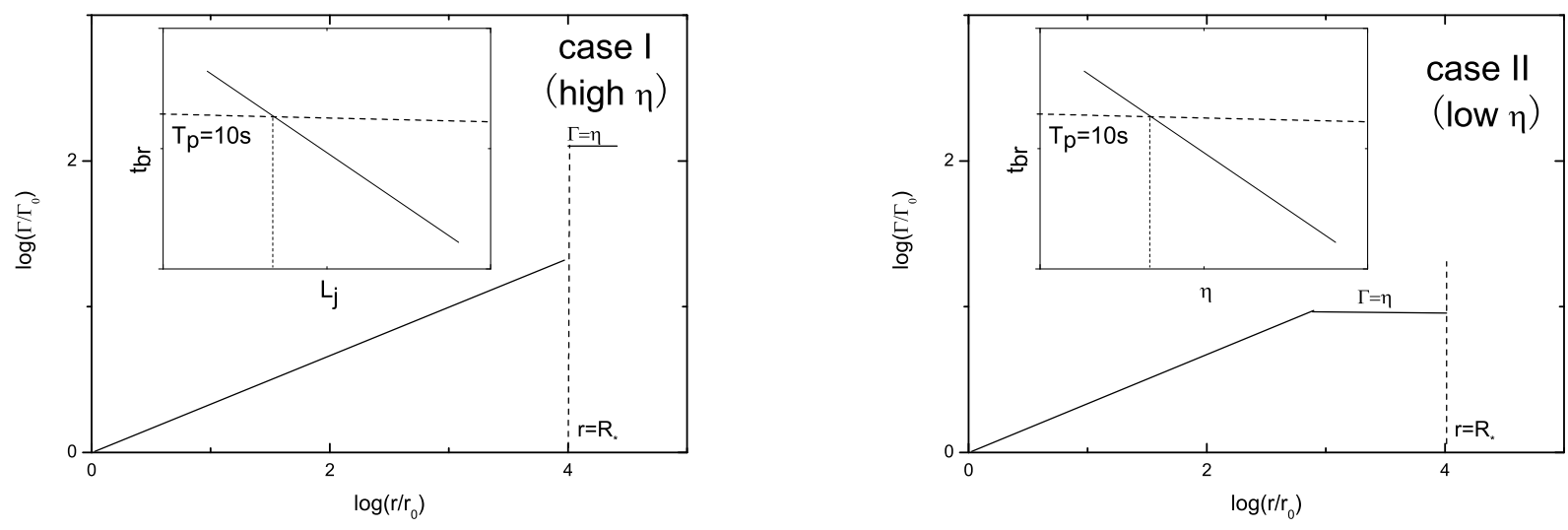

FIG. 1. - The evolution of the jet Lorentz factor with radius for the two different cases defined in the text. The left panel is the high entropy case, where the jet Lorentz factor is not saturated before breaking out of the star and the right one is the lower entropy case, where the jet Lorentz factor has already saturated before breaking out. The inset figures in the two panels show the dependence of the jet breakout time $t_{\mathrm{br}}$ on other parameters for the two cases respectively. Here $r_{0}$ is the initial injection radius, $\Gamma_{0}$ is the initial injection Lorentz factor and $R_{*}$ is the stellar radius of the progenitor. 